Research Article

\title{
Evaluation of Chest CT Scan as a Screening and Diagnostic Tool in Trauma Patients with Coronavirus Disease 2019 (COVID-19): A Cross-Sectional Study
}

\author{
Hossein Abdolrahimzadeh Fard (D), ${ }^{1}$ Salahaddin Mahmudi-Azer ${ }^{(D)}{ }^{2}$ \\ Sepideh Sefidbakht $\mathbb{D}^{3},{ }^{3}$ Pooya Iranpour $\mathbb{D}^{3},{ }^{3}$ Shahram Bolandparvaz, ${ }^{1}$ \\ Hamid Reza Abbasi $\mathbb{D}^{4},{ }^{4}$ Shahram Paydar ${ }^{(D)},{ }^{1}$ Golnar Sabetian $\left(\mathbb{D},{ }^{5}\right.$ \\ Mohamad Mahdi Mahmoudi (D), ${ }^{1}$ Masoume Zare $\left(\mathbb{D},{ }^{1}\right.$ Leila Shayan $\left(\mathbb{D},{ }^{1}\right.$ \\ and Maryam Salimi ${ }^{6}$ \\ ${ }^{1}$ Trauma Research Center, Department of Surgery, Shahid Rajaee (Emtiaz) Trauma Hospital, \\ Shiraz University of Medical Sciences, Shiraz, Iran \\ ${ }^{2}$ Pulmonary Research Group, Department of Medicine, University of Alberta, Edmonton, Alberta, Canada \\ ${ }^{3}$ Medical Imaging Research Center, Department of Radiology, Shiraz University of Medical Sciences, Shiraz, Iran \\ ${ }^{4}$ Education Development and Research Center, Shiraz University of Medical Sciences, Shiraz, Iran \\ ${ }^{5}$ Shiraz Anesthesiology and Critical Care Research Center, Shiraz University of Medical Sciences, Shiraz, Iran \\ ${ }^{6}$ Student Research Committee, Shiraz University of Medical Sciences, Shiraz, Iran
}

Correspondence should be addressed to Maryam Salimi; salimimaryam7496@gmail.com

Received 13 April 2021; Revised 29 April 2021; Accepted 14 June 2021; Published 30 June 2021

Academic Editor: Roberto Cirocchi

Copyright (c) 2021 Hossein Abdolrahimzadeh Fard et al. This is an open access article distributed under the Creative Commons Attribution License, which permits unrestricted use, distribution, and reproduction in any medium, provided the original work is properly cited.

\begin{abstract}
Background. The lack of enough medical evidence about COVID-19 regarding optimal prevention, diagnosis, and treatment contributes negatively to the rapid increase in the number of cases globally. A chest computerized tomography (CT) scan has been introduced as the most sensitive diagnostic method. Therefore, this research aimed to examine and evaluate the chest CT scan as a screening measure of COVID-19 in trauma patients. Methods. This cross-sectional study was conducted in Rajaee Hospital in Shiraz from February to May 2020. All patients underwent unenhanced CT with a 16-slice CT scanner. The CT scans were evaluated in a blinded manner, and the main CT scan features were described and classified into four groups according to RSNA recommendation. Subsequently, the first two Radiological Society of North America (RSNA) categories with the highest probability of COVID-19 pneumonia (i.e., typical and indeterminate) were merged into the "positive CT scan group" and those with radiologic features with the least probability of COVID-19 pneumonia into "negative CT scan group." Results. Chest CT scan had a sensitivity of $68 \%$, specificity of $56 \%$, positive predictive value of $34.8 \%$, negative predictive value of $83.7 \%$, and accuracy of $59.3 \%$ in detecting COVID-19 among trauma patients. Moreover, for the diagnosis of COVID-19 by CT scan in asymptomatic individuals, a sensitivity of $100 \%$, specificity of $66.7 \%$, and negative predictive value of $100 \%$ were obtained ( $p$ value: 0.05 ). Conclusion. Findings of the study indicated that the CT scan's sensitivity and specificity is less effective in diagnosing trauma patients with COVID-19 compared with nontraumatic people.
\end{abstract}

\section{Background}

In March 2020, the World Health Organization (WHO) declared COVID-19 a pandemic. Although it has been nine months into the novel coronavirus disease 2019 (COVID-19) pandemic, the high number of daily cases still remains problematic [1,2]. By September 16, 2020, almost 30 million cases of COVID-19 had been confirmed by laboratory tests, including about 1 million deaths $[3,4]$. Mortality rates were reported as $8 \%$ in the south of Iran, the country that is 
considered one of the most major focal points of the world's disease. It has also had a substantial effect on mental health [5-7]. Although its worldwide mortality rate is less than $4 \%$ today, the lack of definitive treatment [8] makes the diagnosis of infected people to break the disease chain the primary method of dealing with the disease. The foregoing issue necessitates a faster diagnostic method of suspected individuals with definitive diagnostic tests. A large number of asymptomatic carriers have led to greater efforts to identify asymptomatic patients. Concerning the low sensitivity and time-consuming nature of the current serological tests, efforts have been focused on using faster and more sensitive diagnostic tests [9]. The use of a chest CT scan has been found as a promising screening method in asymptomatic patients [10]. Moreover, by improving the definitive diagnostic methods and comparing the findings of chest CT scans in the definitely infected patients, it became clear that chest CT scans can be used with high sensitivity in diagnosing the patients [11]. Even though the Radiology Society of North America (RSNA) has standardized chest CT scan reports, its low negative predictive value has overshadowed the use of CT scans as a convenient screening method [12]. Likewise, chest CT scan is not recommended as a screening tool for asymptomatic patients not only due to the resources required and unnecessary radiation to patients but also because of the low predictive value. Furthermore, while chest CT has a relatively high sensitivity, the specificity is relatively low, with COVID-19 pneumonia exhibiting overlapping features with numerous other diseases; CT cannot be used as a form of diagnosis.

Trauma centers, due to the nature of the patients referred to them and the time constraints they face due to the priority of treating life-threatening injuries, need to perform treatments that cause close contact with the patient and are particularly challenging when dealing with potential COVID-19 patients. Given the extensive use of CT scan as the gold standard diagnostic method in most trauma centers, its availability, and short time to perform, this retrospective study evaluates the sensitivity and specificity of chest CT scan in the diagnosis of COVID-19 in trauma patients. This study investigated the effect of trauma on the clinical findings of CT scans in COVID-19 based on RSNA classification. We also aimed to evaluate the utility of chest CT to help triage trauma patients for potential presence of COVID-19 infection.

\section{Methods}

2.1. Patients and Data Source of RT-PCR Results. This crosssectional study was conducted in Rajaee Hospital in Shiraz, the only dedicated trauma center in Iran with an annual referral rate of 15,000 to 20,000 patients, from February to May 2020. Trauma patients who needed to undertake a chest CT scan for their trauma were included in the study. These patients have been checked for COVID-19 at the time of hospitalization, based on the Quality Improvement Committee's approved protocol. Data regarding the vital signs, submitted tests, and COVID-19-related history have been completed from the medical record system. Patients who did not have a CT scan and RT-PCR test at the time of their arrival or the interval between them was more than 24 hours were excluded from the study. Those who had lung-related underlying disease such as chronic obstructive pulmonary disease (COPD) and lung cancer were also excluded. Clinical symptoms and epidemiological risk factors that were associated with COVID-19 and the underlying diseases were obtained.

This study used a correlational design to examine the relationship between chest CT scan finding and RT-PCR result as the golden standard for definite COVID-19 diagnosis. Patients with a negative RT-PCR test have been rechecked using the lower respiratory tract after four days.

2.2. Chest CT Protocol. All CT scans were obtained with one CT system. All patients underwent unenhanced CT with a 16-slice CT scanner (CT emotion 16, Siemens Healthcare, Erlangen, Germany). Given the probability of COVID-19, all necessary protective measures have been taken according to the Iranian Ministry of Health guidelines [13].

2.3. Image Analysis. Two radiologists with Iranian Board Certification (SS and PI), who were blinded to RT-PCR result and COVID-19-related symptoms, reported all CT scans by consensus. They described main CT scan features (ground-glass opacity (GGO), consolidation, bronchial distortion, reticular line, crazy paving sign, atoll sign, cavity, rib fracture, pneumothorax, hemothorax, diaphragm injury, and shape and pattern of the lesions) and classified the patients into four groups according to RSNA recommendation [14] (Table 1). A chest CT scan is considered to be the most available diagnostic modality in the setting of chest trauma. Therefore, we hypothesized the possibility of utilizing it as an initial screening test for possible identification of the concomitant COVID-19 pneumonia in the trauma cases and also for the proper allocation of the patients to COVID-19 and non-COVID-19 wards before the RT-PCR tests were processed. Therefore, we merged the first two RSNA categories with the highest probability of COVID-19 pneumonia (i.e., typical and indeterminate) into the "positive CT scan group" and those with radiologic features with the least probability of COVID-19 pneumonia into the "negative CT scan group."

2.4. Statistical Analysis. The means and standard deviations were calculated for quantitative variables, and count and percentage were calculated for qualitative variables. A comparison between the two groups was performed using the chi-square test or Fisher's exact test. Moreover, sensitivity and specificity have been calculated to determine the correct diagnosis of different protocols. SPSS version 16 (IBM, United States) was used for statistical analysis. The significance level was considered at 0.05 . 
TABLE 1: RSNA criteria for COVID-19 pneumonia (adopted from reference [14]).

\begin{tabular}{lcr}
\hline $\begin{array}{l}\text { COVID-19 RSNA } \\
\text { classification }\end{array}$ & Rationale & Chest CT scan findings \\
Typical appearance & $\begin{array}{c}\text { Commonly reported imaging feature of } \\
\text { greater specificity for COVID-19 }\end{array}$ & $\begin{array}{c}\text { (i) Peripheral and bilateral GGO with/without consolidation } \\
\text { (ii) Multifocal GGO with/without crazy paving sign } \\
\text { (iii) Reverse halo sign }\end{array}$ \\
\hline $\begin{array}{l}\text { Indeterminate feature } \\
\text { Nonspecific image for COVID-19 }\end{array}$ & $\begin{array}{c}\text { (i) Absence of typical feature } \\
\text { (ii) Presence of multifocal, diffuse, prehilar, or unilateral GGO with/ } \\
\text { without nonround, nonperipheral consolidation }\end{array}$ \\
& (iii) Few very small GGO, nonround and nonperipheral distribution
\end{tabular}

(i) Absence of typical and atypical features

Atypical appearance Uncommonly or not reported feature of COVID-19 (ii) Presence of single lobar or segmental consolidation, discrete small nodules, lung cavitation, and smooth interlobular septal thickening with pleural effusion
Normal for

pneumonia (iii) No CT features to suggest the pneumonia

\section{Results}

Of 842 PCR tests performed, 132 cases were screened on admission according to the hospital guidelines with 30 positive results. According to the following chart, 86 patients (male: $82.6 \%$; female: $17.4 \%$; mean age: $40.5 \pm 20.4$ ) underwent a chest CT scan according to the trauma mechanism, and the surgical guidelines were included in the study (Figure 1).

Typical and indeterminate reports were classified as positive CT scans for COVID-19, while atypical and normal CT scans were reported as a negative group. According to the outcomes of CT scan, 44 patients (male: 85.7\%; female: $14.3 \%$ ) had typical and indeterminate findings for COVID19 and 42 patients (male: $79.5 \%$; female: $20.5 \%$ ) had atypical or normal CT finding. The analysis revealed no significant statistical difference between the two groups on age, sex, and history of underlying diseases (Table 2). Comparison of race, clinical symptoms, and epidemiological risk factors associated with COVID-19 between positive and negative groups showed that the Iranian race $(97.7 \%, 81 \%$, PV: 0.014$)$, incidence of acute respiratory symptoms (63.9\%, 36.1\%, PV: $0.037)$, and history of dyspnoea $(38.6 \%, 16.7 \%$, PV: 0.02$)$ had significant differences in the existence between the two types of CT scan groups. In correlation with the RT-PCR result, $31.8 \%$ definite patients had a negative chest CT scan, whereas $100 \%$ of them were likely to be infected by COVID-19 and $28.3 \%$ were highly suspicious for COVID-19. In the patients with the negative RT-PCR result, $43.7 \%$ had positive chest CT scan findings, of which $75 \%$ could be infected by COVID-19.

Patients were divided into four groups according to different RSNA categories. According to RSNA classification, the chest CT scans were reported as typical (26.7\%), indeterminate (24.4\%), atypical (29.1\%), and normal $(19.8 \%)$. In correlation with RT-PCR result in definite COVID-19 group, incidence of RSNA type (T/I/A/N) was $31.8 \%, 36.4 \%, 27.3 \%$, and $4.5 \%$. In a typical group, $69.6 \%$ had negative RT-PCR and $30.4 \%$ had positive RT-PCR results. In patients with a report of normal CT scan, $5.9 \%$ had a positive RT-PCR result.

There was a significant difference between the four groups of RSNA (T, I, A, N) regarding the mean of age (48.7, 36.8, 43.6, and 30.3; PV: 0.023), Iranian nationality (95.7, 100, 86.2, and 77.8; PV: 0.023), and incidence of acute respiratory symptoms $(52.2,45,87$, and 44.4 ; PV: 0.012$)$. None of the respiratory symptoms were significantly different between the four groups.

Based on the RT-PCR test results, patients were divided into two groups of definite and noninfected patients, and based on the diagnostic and nondiagnostic type of CT scan and RSNA recommendation classes, different features, sensitivity, specificity, positive predictive value, negative predictive value, and accuracy of them were assessed for a diagnosis of definite patients. Incidence of different common COVID-19 features in chest CT scan was investigated in these two groups (Table 3).

The sensitivity and specificity of the positive CT group, typical RSNA type, and different features of the CT scan report were calculated as shown in Table 4.

Positive chest CT scans for COVID-19 were compared between two groups according to RT-PCR results. Fifteen patients with positive RT-PCR test and 28 patients with negative RT-PCR results had positive chest CT scans features for COVID-19. So, a chest CT scan had a sensitivity of $68 \%$, specificity of $56 \%$, positive predictive value of $34.8 \%$, negative predictive value of $83.7 \%$, and accuracy of $59.3 \%$ in detecting COVID-19 among trauma patients.

Evaluation of 23 asymptomatic patients (two with positive RT-PCR and 21 negative RT-PCR results) showed that all definitively infected patients have a positive CT scan for the disease and only seven noninfected patients have a positive CT scan. Statistical analysis showed a sensitivity of $100 \%$ and specificity of $66.7 \%$ and negative predictive value of $100 \%$ for the diagnosis of COVID-19 by CT scan in asymptomatic individuals. 


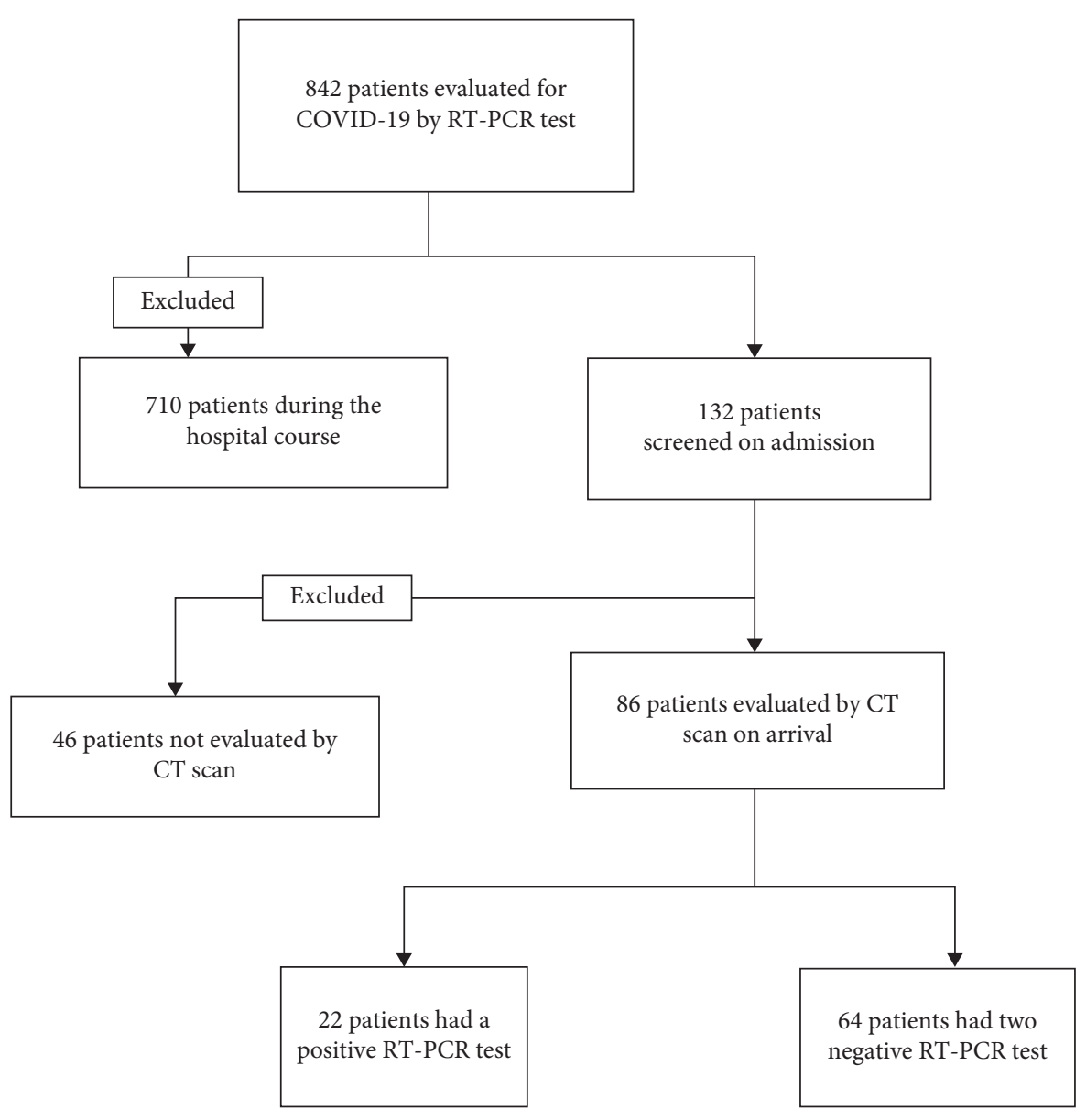

Figure 1: Pathway design of the study.

TABLe 2: Comparison of COVID-19-related criteria between positive and negative CT scan groups.

\begin{tabular}{|c|c|c|c|}
\hline Variables & Chest CT scan +ve (\%) $n=44$ & Chest CT scan -ve (\%) $n=42$ & $p$ value \\
\hline \multicolumn{4}{|l|}{ Nationality } \\
\hline Iranian & 97.7 & 81 & 0.014 \\
\hline Non-Iranian & 2.3 & 19 & \\
\hline \multicolumn{4}{|c|}{ Positive COVID-19-related history } \\
\hline Contact & 6.8 & 14.3 & 0.3 \\
\hline Fever & 27.1 & 38.1 & 0.2 \\
\hline Cough & 13.6 & 11.9 & 0.53 \\
\hline Respiratory distress & 38.6 & 16.7 & 0.02 \\
\hline Neurological symptoms & 15.9 & 4.8 & 0.08 \\
\hline Dermatological symptoms & 0 & 0 & - \\
\hline Abdominal pain & 2.3 & 2.4 & 1 \\
\hline Anorexia & 6.8 & 2.4 & 0.61 \\
\hline \multicolumn{4}{|l|}{ Positive past medical history } \\
\hline Cancer & 0 & 2.4 & 0.48 \\
\hline Diabetes mellitus & 9.1 & 7.1 & 1 \\
\hline Chronic anemia & 0 & 2.4 & 0.48 \\
\hline Cardiac disease & 9.1 & 11.9 & 0.73 \\
\hline Chronic renal failure & 2.3 & 0 & 1 \\
\hline Asthma & 2.3 & 0 & 1 \\
\hline COPD & 2.3 & 2.4 & 1 \\
\hline HTN & 9.1 & 4.8 & 0.67 \\
\hline
\end{tabular}


TABLE 2: Continued.

\begin{tabular}{|c|c|c|c|}
\hline Variables & Chest CT scan +ve (\%) $n=44$ & Chest CT scan -ve (\%) $n=42$ & $p$ value \\
\hline \multicolumn{4}{|l|}{ Hospital course } \\
\hline Intubation need & 52.3 & 35.7 & 0.09 \\
\hline ICU admission & 77.3 & 61.9 & 0.09 \\
\hline ARDS incidence & 39 & 41.7 & 0.75 \\
\hline Mortality & 25 & 9.5 & 0.53 \\
\hline \multicolumn{4}{|l|}{ Patient classification } \\
\hline Suspicious (WHO) & 2.3 & 4.8 & 0.61 \\
\hline Possible (ECDC) & 79.5 & 66.7 & 0.13 \\
\hline Asymptomatic & 20.5 & 33.3 & 0.2 \\
\hline
\end{tabular}

CT: computed tomography; COVID-19: coronavirus disease 2019; COPD: chronic obstructive pulmonary disease; ARDS: acute respiratory distress syndrome; HTN: hypertension; ICU: intensive care unit; WHO: World Health Organization; ECDC: European Centre for Disease Prevention and Control.

TABLe 3: Incidence of different RSNA classification features in definite and noninfected patients.

\begin{tabular}{|c|c|c|}
\hline Finding & RT-PCR ${ }^{* *}$ positive $(\%) n=22$ & RT-PCR negative (\%) $n=64$ \\
\hline \multicolumn{3}{|l|}{$G G O^{*}$} \\
\hline Presence & $15(68.1)$ & $30(46.8)$ \\
\hline Multiple bilateral & $10(45.4)$ & $22(34.3)$ \\
\hline Multiple unilateral & $2(9)$ & $2(3.1)$ \\
\hline Single unilateral & $3(13.6)$ & $6(9.3)$ \\
\hline Peripheral & $11(50)$ & $13(20.3)$ \\
\hline Central & $2(9)$ & $4(6.2)$ \\
\hline Peripheral/central & $2(9)$ & $13(20.3)$ \\
\hline Irregular & $9(40.3)$ & $25(39)$ \\
\hline Round & $3(13.6)$ & $2(3.1)$ \\
\hline Round/irregular & $3(13.6)$ & $3(4.6)$ \\
\hline \multicolumn{3}{|l|}{ Consolidation } \\
\hline Presence & $11(50)$ & $22(34.3)$ \\
\hline Multiple bilateral & $8(36.3)$ & $13(20.3)$ \\
\hline Multiple unilateral & - & - \\
\hline Single unilateral & $3(13.6)$ & $9(14)$ \\
\hline Peripheral & $8(36.3)$ & $17(26.5)$ \\
\hline Central & $2(9)$ & $1(1.5)$ \\
\hline Peripheral/central & $1(4.5)$ & $4(6.2)$ \\
\hline Irregular & $9(40.9)$ & $18(28.1)$ \\
\hline Round & - & - \\
\hline Round/irregular & $2(9)$ & $4(6.2)$ \\
\hline \multicolumn{3}{|l|}{ Rib fracture } \\
\hline Presence & $3(13.6)$ & $14(21.8)$ \\
\hline With pneumothorax & $3(13.6)$ & $6(9.3)$ \\
\hline With pleural effusion & $2(9)$ & $8(12.5)$ \\
\hline \multicolumn{3}{|l|}{ Pneumothorax } \\
\hline Presence & $7(31.8)$ & $10(15.6)$ \\
\hline Without rib fracture & $4(18.1)$ & $4(6.2)$ \\
\hline \multicolumn{3}{|l|}{ Hemothorax } \\
\hline Presence & $11(50)$ & $15(23.4)$ \\
\hline Without rib fracture & $9(40.9)$ & $7(10.9)$ \\
\hline
\end{tabular}

*GGO: ground-glass opacity. ${ }^{* *}$ RT-PCR: reverse transcription polymerase chain reaction.

\section{Discussion}

The early diagnosis of COVID-19 plays an important role in monitoring the disease effectively. According to the initial definition provided by the World Health Organization, patients with suspected clinical symptoms should be evaluated by RT-PCR testing for COVID-19 [15]. Related antibodies need to be evaluated for definitive COVID-19, which is conducted by evaluating the presence of the virus in different ways. The results of these methods are related to different factors such as the time of exposure to the virus and the onset of symptoms, which are effective in positive serological tests $[9,16]$. The diagnosis of asymptomatic patients and patients with mild symptoms, which includes a high percentage of patients [17], is also an important goal of healthcare organizations. Taken into consideration the low sensitivity of definitive diagnostic tests (RT-PCR) [9, 18], other diagnostic methods are used to accompany them 
TABle 4: Diagnostic values of computed tomography findings in COVID-19 trauma patients.

\begin{tabular}{|c|c|c|c|c|c|c|}
\hline Finding & Sensitivity (\%) & Specificity (\%) & $\begin{array}{l}\text { Positive predictive value } \\
(\%)\end{array}$ & $\begin{array}{c}\text { Negative predictive value } \\
(\%)\end{array}$ & Accuracy (\%) & $p$ value \\
\hline \multicolumn{7}{|l|}{$\overline{G G O^{*}}$} \\
\hline Presence & 68 & 53 & 33 & 82 & 56 & 0.05 \\
\hline Multiple bilateral & 45 & 65 & 31 & 77 & 60 & 0.55 \\
\hline Multiple unilateral & 9 & 96 & 50 & 75 & 74 & 0.83 \\
\hline Single unilateral & 13 & 90 & 33 & 75 & 70 & 0.79 \\
\hline Peripheral & 50 & 79 & 45 & 82 & 72 & 0.13 \\
\hline Central & 9 & 93 & 33 & 75 & 72 & 0.9 \\
\hline Peripheral/central & 9 & 79 & 13 & 71 & 61 & 0.71 \\
\hline Irregular & 40 & 60 & 26 & 75 & 55 & 0.92 \\
\hline Round & 13 & 96 & 60 & 76 & 75 & 0.72 \\
\hline Irregular/round & 13 & 95 & 50 & 76 & 74 & 0.73 \\
\hline \multicolumn{7}{|l|}{ Consolidation } \\
\hline Presence & 50 & 65 & 33 & 79 & 61 & 0.39 \\
\hline Multiple bilateral & 36 & 79 & 38 & 78 & 68 & 0.43 \\
\hline Single unilateral & 13 & 85 & 25 & 74 & 67 & 0.98 \\
\hline Peripheral & 36 & 73 & 32 & 77 & 63 & 0.62 \\
\hline Central & 9 & 98 & 66 & 75 & 75 & 0.83 \\
\hline Peripheral/central & 4 & 93 & 20 & 74 & 70 & 0.95 \\
\hline Irregular & 40 & 71 & 33 & 77 & 63 & 0.51 \\
\hline Irregular/round & 9 & 93 & 33 & 75 & 72 & 0.9 \\
\hline \multicolumn{7}{|l|}{ Rib fracture } \\
\hline Presence & 13 & 78 & 17 & 72 & 61 & 0.76 \\
\hline $\begin{array}{l}\text { With } \\
\text { pneumothorax }\end{array}$ & 13 & 90 & 33 & 75 & 70 & 0.85 \\
\hline With plural effusion & 9 & 87 & 20 & 73 & 67 & 0.89 \\
\hline \multicolumn{7}{|l|}{ Pneumothorax } \\
\hline Presence & 31 & 84 & 41 & 78 & 70 & 0.44 \\
\hline Without rib fracture & 18 & 93 & 50 & 76 & 74 & 0.63 \\
\hline \multicolumn{7}{|l|}{ Hemothorax } \\
\hline Presence & 50 & 76 & 42 & 81 & 69 & 0.16 \\
\hline Without rib fracture & 40 & 89 & 56 & 81 & 76 & 0.19 \\
\hline
\end{tabular}

${ }^{*}$ GGO: ground-glass opacity.

[19-21]. Typical chest CT scan findings in people with definite COVID-19 pneumonia (Table 1) are one of the most commonly used methods. This study investigated the effectiveness of RSNA classification and its features as a diagnostic tool for COVID-19 in trauma patients. Due to the presence of similar manifestations of lung contusion in chest CT scan and also the possibility of simultaneous occurrence of these two pathologies, their interactions on CT scan findings must be evaluated in trauma patients [22]. Evaluation of chest CT scan findings based on RSNA classification showed the lower sensitivity and specificity among the trauma patients. This outcome can be related to the prevalence rate of the disease, low sensitivity of the RT-PCR diagnostic test, and the weakness of the chest CT scan findings, which attributed to infected nontraumatic patients for traumatic individuals.

Given the high prevalence of the disease in the area where the study was conducted and the acceptable sensitivity of the diagnostic tests, the findings of the chest CT scan for COVID-19 as a diagnostic tool might differ among trauma patients.

Patients were divided into positive and negative groups based on their chest CT scan findings, regardless of RT-PCR serological test results. Evaluation of clinical symptoms related to COVID-19, systemic inflammatory response syndrome (SIRS) index and lymphocyte-to-neutrophil ratio (NLR) as a marker of systemic inflammatory responses, factors related to patients' trauma including primary vital signs as well as injury severity score (ISS) and abbreviated injury score (AIS), patient's hospital course, and prognosis of patients between the two groups was performed. The evaluation showed that patients were not significantly different in terms of age, COVID-19-related symptoms (except for respiratory distress), and epidemiological and underlying disease history. The evaluation of inflammatory and immune system stimulation factors as well as criteria related to the severity of trauma did not show a significant difference between the two groups. With the exception of race and respiratory distress, factors related to COVID-19 and inflammatory system and trauma severity do not affect the probability of positive chest CT scan according to the criteria provided by RSNA.

The outcomes of this research are not in line with studies investigating nontraumatic individuals [23]. Trauma and COVID-19 both stimulate the immune system. However, the degree of stimulation of the inflammatory system 
depends on the degree of trauma severity and also the inflammatory stage of COVID-19. It is hypothesized that if the criteria set by RSNA were appropriate for the evaluation of COVID-19 traumatic individuals, a significant difference should be observed between the two groups.

Evaluation of common radiological features based on RSNA classification between the two groups of definitive and noninfected patients showed that the presence of peripheral, bilateral, round, and diffuse GGO as the most specific radiological features in nontraumatic individuals [24] is not similar in traumatic patients. Although multiple bilateral GGO was the most sensitive in lesions (45\%) according to the location in trauma patients, the specificity of multiple unilateral lesions was higher (96\%). Moreover, the peripheral lesions were more sensitive and the central lesions had the highest specificity (93\%). In terms of lesion shape, the highest sensitivity was related to irregular lesions (40\%) and the highest specificity was related to round lesions (96\%). Therefore, multiple unilateral or central GGO in trauma patients was the most specific type of GGO in trauma patients who had definite COVID-19. Regarding consolidation, peripheral round lesions had the most diagnostic features in nontraumatic patients, but central lesions (98\%) with mixed round and irregular shapes had the highest diagnostic features in traumatic patients with COVID-19.

Despite the previously mentioned data, due to the possibility of co-occurrence of COVID-19 and pulmonary contusion in both groups, the findings of this research should be treated with caution and future studies should collect information about trauma patients in the prepandemic period of COVID-19 to determine specific lung contusion lesions.

Therefore, it seems that, for the detection of COVID-19 in trauma patients, it is necessary to provide another classification for a chest CT scan. This requires further studies with a larger population and also the use of more sensitive definitive diagnostic tests.

In the evaluation of 17 patients with rib fractures in both definite and noninfected patients, it was found that rib fracture in $100 \%$ of definite patients and $71 \%$ noninfected patients leads to damage to the pulmonary parenchyma and pleura. Also, patients with less ISS in a definite group develop more symptoms of regional injury in the thoracic cavity (AIS). This indicates that the presence of underlying pathology due to COVID-19 in traumatic individuals causes the lung parenchyma to be prone to injury and rupture. Moreover, it seems that the ISS/thoracic cavity AIS ratio may contribute to the possibility of underlying pathology in traumatized individuals. However, due to the small number of participants, it was not possible to statistically evaluate this finding further. There were some limitations in our study. It is possible that patients could not remember the associated symptom of COVID-19 or the history of contact with a suspicious person. Further multicentric studies with a large sample size were essential to find the complete disadvantages of using the routine criteria in the trauma patient. Overall, the results of this study can be the basis for further studies to introduce a new triage system and criteria in trauma patients.

\section{Conclusion}

The results show that RSNA criteria for COVID-19 were not efficient in trauma patients. Therefore, due to the high use of CT scans in trauma patients, it is recommended to create appropriate CT scan criteria for trauma patients. This method could diagnose the disease timely and contribute positively to the termination of the transmission chain. It would also reduce the incidence of treatment and effective resource management.

\section{Abbreviations \\ CT: $\quad$ Computed tomography \\ ICU: Intensive care unit \\ COVID-19: Coronavirus disease 2019 \\ COPD: Chronic obstructive pulmonary disease \\ RSNA: Radiological Society of North America \\ WHO: World Health Organization \\ RT-PCR: Reverse transcription polymerase chain reaction \\ GGO: $\quad$ Ground-glass opacity \\ SIRS: $\quad$ Systemic inflammatory response syndrome \\ NLR: $\quad$ Lymphocyte-to-neutrophil ratio \\ ISS: Injury severity score \\ AIS: $\quad$ Abbreviated injury score.}

\section{Data Availability}

SPSS data of the participants are available upon reasonable request from the corresponding author.

\section{Ethical Approval}

This study was performed in line with the principles of the Declaration of Helsinki. Approval was granted by the Ethical Committee of Shiraz University of Medical Sciences.

\section{Consent}

Written informed consent was obtained from all individual participants included in the study. The purpose of this research was completely explained to the patients and they were assured that their information will be kept confidential by the researcher. A copy of the written consent is available for review by the editor of this journal.

\section{Disclosure}

A preprint copy of the article is available in Research Square (DOI: 10.21203/rs.3.rs-110402/v2).

\section{Conflicts of Interest}

All authors certify that they have no affiliations with or involvement in any organization or entity with any financial interest or nonfinancial interest in the subject matter or materials discussed in this manuscript. 


\section{Authors' Contributions}

HAF conceived the idea for the manuscript and in cooperation with SB and SP interpreted the patient data and carried out the treatment. SS and PI reported the chest CT scans. MM collected the data and drafted the manuscript. MZ and GS contributed to data collection. HRA and LS analysed the data. MS and SMA revised the manuscript and acted as guarantors of the manuscript. All authors read and approved the final manuscript.

\section{References}

[1] L. Chen, J. Xiong, L. Bao, and Y. Shi, "Convalescent plasma as a potential therapy for COVID-19," The Lancet Infectious Diseases, vol. 20, no. 4, pp. 398-400, 2020.

[2] S. Meshkat, A. Salimi, A. Joshaghanian, S. Sedighi, S. Sedighi, and V. Aghamollaii, "Chronic neurological diseases and COVID-19: associations and considerations," Translational Neuroscience, vol. 11, no. 1, pp. 294-301, 2020.

[3] Anadolu Agency, "Confusion raises Iranian fears from coronavirus 2020," 2020, https://www.aa.com.tr/en/health/ confusion-raises-iranian-fears-from-coronavirus/1745093.

[4] BNO News, "World tracking Coronavirus: Map, Data and Timeline 2020," 2020, https://bnonews.com/index.php/2020/ 02/the-latest-coronavirus-cases/.

[5] A. Erfani, R. Shahriarirad, K. Ranjbar, A. Mirahmadizadeh, and M. Moghadami, "Knowledge, attitude and practice toward the novel coronavirus (COVID-19) outbreak: a population-based survey in Iran," BMC Infectious Disease, vol. $427,2020$.

[6] A. Ammar, K. Trabelsi, M. Brach et al., "Effects of home confinement on mental health and lifestyle behaviours during the COVID-19 outbreak: insights from the ECLB-COVID19 multicentre study," Biology of Sport, vol. 38, no. 1, pp. 9-21, 2021.

[7] A. Ammar, H. Chtourou, O. Boukhris et al., "COVID-19 home confinement negatively impacts social participation and life satisfaction: a worldwide multicenter study," International Journal of Environmental Research and Public Health, vol. 17, no. 17, p. 6237, 2020.

[8] A. Ammar, P. Mueller, K. Trabelsi et al., "Psychological consequences of COVID-19 home confinement: the ECLBCOVID19 multicenter study," PLoS One, vol. 15, no. 11, Article ID e0240204, 2020.

[9] S. Inui, A. Fujikawa, M. Jitsu et al., "Erratum: chest CT findings in cases from the cruise ship "diamond princess" with coronavirus disease 2019 (COVID-19)," Radiology: Cardiothoracic Imaging, vol. 2, no. 2, Article ID e204002, 2020.

[10] M. Sadeghi, P. Saberian, P. Hasani-Sharamin, F. Dadashi, S. Babaniamansour, and E. Aliniagerdroudbari, "The role of possible factors affecting the risk of getting infected by COVID-19 in emergency medical technicians: a case-control study," Research Square, 2020.

[11] M. Lotfi, S. Sefidbakht, M. Moghadami et al., "Introduction of a radiologic severity index for the 2019 novel corona virus (COVID-19)," Research Square, 2020.

[12] H. Kim, H. Hong, and S. H. Yoon, "Diagnostic performance of $\mathrm{CT}$ and reverse transcriptase polymerase chain reaction for coronavirus disease 2019: a meta-analysis," Radiology, vol. 296, no. 3, pp. E145-E155, 2020.
[13] A. Raeisi, J. S. Tabrizi, and M. M. Gouya, "IR of Iran national mobilization against COVID-19 epidemic," Archives of Iranian Medicine, vol. 23, no. 4, pp. 216-219, 2020.

[14] S. Simpson, F. U. Kay, S. Abbara et al., "Radiological society of North America expert consensus statement on reporting chest CT findings related to COVID-19. Endorsed by the society of thoracic radiology, the American college of radiology, and RSNA-secondary publication," Journal of Thoracic Imaging, vol. 35, no. 4, pp. 219-227, 2020.

[15] World Health Organization, Clinical Management of COVID19: Interim Guidance, World Health Organization, Geneva, Switzerland, 2020, https://apps.who.int/iris/handle/10665/ 332196.

[16] L. M. Kucirka, S. A. Lauer, O. Laeyendecker, D. Boon, and J. Lessler, "Variation in false-negative rate of reverse transcriptase polymerase chain reaction-based SARS-CoV-2 tests by time since exposure," Annals of Internal Medicine, vol. 173, no. 4, pp. 262-267, 2020.

[17] World Health Organization, Coronavirus Disease 2019 (COVID-19): Situation Report, World Health Organization, Geneva, Switzerland, 2020, https://apps.who.int/iris/handle/ $10665 / 331443$.

[18] Y. Yang, M. Yang, C. Shen et al., "Evaluating the accuracy of different respiratory specimens in the laboratory diagnosis and monitoring the viral shedding of 2019-nCoV infections," MedRxiv, vol. 1, 2020.

[19] T. Ai, Z. Yang, H. Hou et al., "Correlation of chest CT and RT-PCR testing for coronavirus disease 2019 (COVID-19) in China: a report of 1014 cases," Radiology, vol. 296, no. 2, pp. E32-E40, 2020.

[20] B. Li, X. Li, Y. Wang et al., "Diagnostic value and key features of computed tomography in Coronavirus Disease 2019," Emerging Microbes \& Infections, vol. 9, no. 1, pp. 787-793, 2020.

[21] K. Li, J. Wu, F. Wu et al., "The clinical and chest CT features associated with severe and critical COVID-19 pneumonia," Investigative Radiology, vol. 55, no. 6, pp. 327-331, 2020.

[22] S. Sefidbakht, S. H. Jafari, F. Zarei et al., "Chest CT scan in patients with contusion and COVID-19, a comparative study with pure lung contusion," Research Square, 2020.

[23] A. Kovács, P. Palásti, D. Veréb, B. Bozsik, A. Palkó, and Z. T. Kincses, "The sensitivity and specificity of chest CT in the diagnosis of COVID-19," Eur. Radiol., pp. 1-6, 2020.

[24] M.-Y. Ng, E. Y. P. Lee, J. Yang et al., "Imaging profile of the COVID-19 infection: radiologic findings and literature review," Radiology: Cardiothoracic Imaging, vol. 2, no. 1, Article ID e200034, 2020. 